Gezeichnete Evidentia 

Iris Brahms (Hrsg.)

\section{Gezeichnete Evidentia}

Zeichnungen auf kolorierten Papieren in Süd und Nord von 1400 bis 1700

mit einem Epilog von Anne Eusterschulte

De Gruyter 
Gedruckt mit freundlicher Unterstützung der Ernst-Reuter-Gesellschaft der Freunde, Förderer und Ehemaligen der Freien Universität Berlin e. V., der Geschwister Boehringer Ingelheim Stiftung für Geisteswissenschaften in Ingelheim am Rhein, des Arbeitskreises Niederländisches Kunst- und Kulturgeschichte e. V. und des Lehrstuhls von Prof. Dr. Anne Eusterschulte.
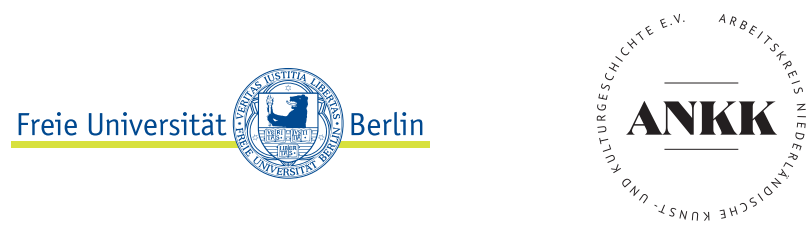

ISBN 978-3-11-063449-5

e-ISBN (PDF) 978-3-11-076075-0

\section{Library of Congress Control Number: 2021949544}

\section{Bibliografische Information der Deutschen Nationalbibliothek}

Die Deutsche Nationalbibliothek verzeichnet diese Publikation in der Deutschen Nationalbibliografie; detaillierte bibliografische Daten sind im Internet über http://dnb.dnb.de abrufbar.

(c) 2022 Walter de Gruyter GmbH, Berlin/Boston

Einbandabbildung: Hendrick Goltzius, Sine Cerere et Libero friget Venus (Detail), ca. 1600, Feder, braune Tinte und Öl auf blaugrau grundierter Leinwand, 105,1 ×80,0 cm, Philadelphia, Philadelphia Museum of Art, Inv. Nr. 1990-100-1 @ Philadelphia Museum of Art

Covergestaltung: Katja Peters, Berlin

Satz: LVD GmbH, Berlin

Druck und Bindung: Beltz Grafische Betriebe GmbH, Bad Langensalza

www.degruyter.com 
